\title{
Cultural Domain in Development and Validation of Tests on Arts to Assess the Skills of Student Graphic Designers
}

\author{
MARIANNA O. NEROZNA ${ }^{1}$, IEVGEN P. GULA ${ }^{1}$, MYKHAILO F. RUDENKO ${ }^{1}$, OKSANA V. \\ MAZNICHENKO $^{1}$, OLEKSANDRA P. BASANEC ${ }^{1}$, VOLODYMYR F. PRUSAK ${ }^{2}$ \\ ${ }^{1}$ Department of Drawing and Painting, Faculty of Design, Kyiv National University of Technologies \\ and Design, Kyiv, UKRAINE \\ ${ }^{2}$ Department of Design, Institute of Woodworking, Computer Technology and Design, Ukrainian \\ National Forestry University, Lviv, UKRAINE
}

\begin{abstract}
The purpose of the study was to examine whether the presence of items that covered cultural questions in the test on arts for student graphic designers influenced the fairness of the test across different ethnic and social groups. The reason for the study was to address the gap in the development and validation of tests on arts that include a cultural domain to assess the skills of undergraduate graphic designers. The research design was based on DIF and DTF methods to examine the factorial structure of test data and to identify whether the factorial structure of the test was the same across ethnic and social groups. A one-factor CFA model was applied to perform measurements for categorised ethnic and social status groups to identify whether the factorial structure was similar or identical for them. The goodness-of-fit measures were calculated using the chi-square statistics, CFI, TLI, and RMSEA to identify how the obtained data is consistent with the hypothesised model. The inclusion of local culture-related questions in the tests on arts to assess the skills of student graphic designers influences the individuals' latent traits which lead to an increase in DIF values. Though there were detected seven items with the DIF for the whole test, the DTF measurements showed that the DIF effect eliminated each out at test level which is related to the fact the certain ethnic groups performed better in answering some specific test items, while certain status groups performed better in answering some other test items. It is noteworthy that DTF for the Ukrainian art (miscellaneous) section was between 0.07 and 0.14 meaning a moderate DTF effect. However, the DTF variance values for the sections of principles of design and principles of art were lower than 0.07 meaning a small effect. Therefore, it could be concluded that small DTF effects found in both the whole test and each test section separately indicated that DIF effects eliminate each other at the test level.
\end{abstract}

Key-Words: - Higher education, student graphic designers, test fairness and validity, students' skills assessment, ethnic and social status bias, differential item functioning, differential test functioning

Received: April 17, 2021. Revised: November 2, 2021. Accepted: December 2, 2021. Published: January 3, 2022.

\section{Introduction}

The development of fair tests on arts containing a cultural domain, specifically the local culture-based one, to assess the skills of student graphic designers is the foremost concern of the relevant university teachers because different student ethnic and social groups perceive cultural questions differently. The concern is related to measurement and cultural biases which are difficult to deal with adequately employing common statistical or classical methods [1-2]. Since the cultural domain relies on latent constructs such as attitudes and perceptions across different groups when common statistical methods are used, there are two causes of measurement bias, first, the item-level bias which is related to variations in answering the question by individuals with the same level of ability but come from different social or ethnic groups, second, the testlevel bias which is associated with dissimilarities in the estimated total scores for the test-takers who are found to be homogeneous in their level of ability but belong to different groups. The item response theory (IRT) methods seem effective in dealing with measurement bias through the use of methods of Differential Item functioning (DIF) and Differential Test Functioning (DTF) because these allow identifying whether the developed test provides valid and reliable (unbiased) results [3-4]. In the literature, the above methods are found to be used for checking the reliability and validity of the tests in languages, tests for social studies like psychometrical or psychological tests [5-8]. However, the study found a gap in the development and validation of tests on arts that include a cultural 
domain to assess the skills of undergraduate graphic designers.

\subsection{Problem Formulation}

The review found that DIF and DTF methods are often used to validate the tests and they showed that DIF and DTF can occur at the domain level, level of the entire test, level of individual and group [9-10]. The methods are based on the item response function concept which is referred to as a mathematical formula that relies on one or more parameters used to identify how the probability of a specific response to a dichotomous question is related to the level of manifestation of a latent trait [4]. Both DIF and DTF methods fall under the Item Response Theory (IRT) which is described in the literature as a direction of a conventional theory of measurement which is based on three constructs such as the factual score, the observed or actual score, and the coefficient of reliability [11]. The IRT models suppose that unidimensionality at both test and test item levels should be considered together with the latter three constructs as there is the assumption that item scores might be affected by the latent constructs [12]. The above concepts are discussed in greater detail by [13-16].

Since cultural and social issues often cause test bias which leads to unreliable results, this inspired this research and proved it to be feasible.

The purpose of the study is to examine whether the presence of items that cover cultural questions in the test on arts for student graphic designers influence the fairness of the test across different ethnic and social groups.

The research questions were as follows: a) whether the factorial structure of the test on arts consisting of cultural questions meets the assumption of unidimensionality before the DIF method is used; b) whether items of the test on arts consisting of cultural questions function differently across ethnic and social groups; c) whether the distribution of DIF items across the cultural subdomain is different; d) whether the entire test scores of the test show differential test functioning (DTF) across ethnic and social groups when each domain is treated as a separate test.

\section{Methods and Materials}

The research design was based on the research questions that were supposed, first, to examine the factorial structure of test data to identify whether the factorial structure of the test was the same across ethnic and social groups. To carry out this, a one- factor CFA model was applied to perform measurements for categorised ethnic and social status groups to identify whether the factorial structure was similar or identical for them. The goodness-of-fit measures were calculated using the chi-square statistics, CFI, TLI, and RMSEA to identify how the obtained data is consistent with the hypothesised model. The items of the test were also examined for facial bias by three experts in linguistic psychology with a Ph.D. degree [2]. When the IRT model was prepared, the test was uploaded to Assess.ai (can be accessed via https://grltd.assess.ai/) which was employed to identify items that exhibited DIF. The reference values were set to be less than 0.05 for the significance level and 9.20 for the detection threshold. Further to this, the differential test functioning (DTF) method (the MantelHaenszel/Liu-Agresti method) was used to identify how the DIF items were related to the test scores that seemed to indicate the unfair assessment [17]. This phase relied on the criteria (reference values) to identify the DTF for the Mantel -Haenszel/LiuAgresti DTF method such as $<0.07$ is a small DTF effect, from 0.07 to 0.14 is a medium DTF effect and $>0.14$ is a large DTF effect [18]. Give the above, the values of $>0.14$ were used as reference ones when calculating DTF statistics.

\subsection{Sampling}

A single-stage cluster sampling technique was used to hire students majoring in graphic design at Kharkiv State Academy of Design and Arts (KSADA), Ukraine; Trans-Carpathian Academy of Arts (TCAA), Ukraine; Kyiv National University of Technologies and Design (KNUTD), Ukraine; Kyiv State Academy of Decorative and Applied Arts and Design named after Mykhailo Boychuk (KSADAAD), Ukraine; and Lviv National Academy of Arts (LNAA), Ukraine. The sampling procedure was organised as a flow of four steps. First, the population of 278 students was defined. This number of student population seemed representative because it was equal to about 20-25 $\%$ of the total number of undergraduates of the graphic design major. Second, the population was divided into clusters of between 50 and 56 people each. Third, the students were informed about the purpose and specifics of the study and they were randomly invited to participate in it. Fourth, those students who agreed formed the cluster-based sample for the study. The number of clusters corresponded to the number of ethnic groups distinguished by nation, religion, culture, and social treatment. The number of subjects in the cluster was 
supposed to be approximately equal. It was between 20-25 students per cluster which aligned with previous research [19-20]. The key inclusion criteria for the test to have been taken were as follows: a respondent left the information about their gender, age, ethnic group (then categorised regionally as
Western Ukrainians, Central and Southern Ukrainians, and Eastern Ukrainians), religious confession belonging (Orthodox, Catholic, Muslim, Other), and social status of their family (categorised as low-income, middle income, and high income).

Table 1. The demographic features of the sampled students

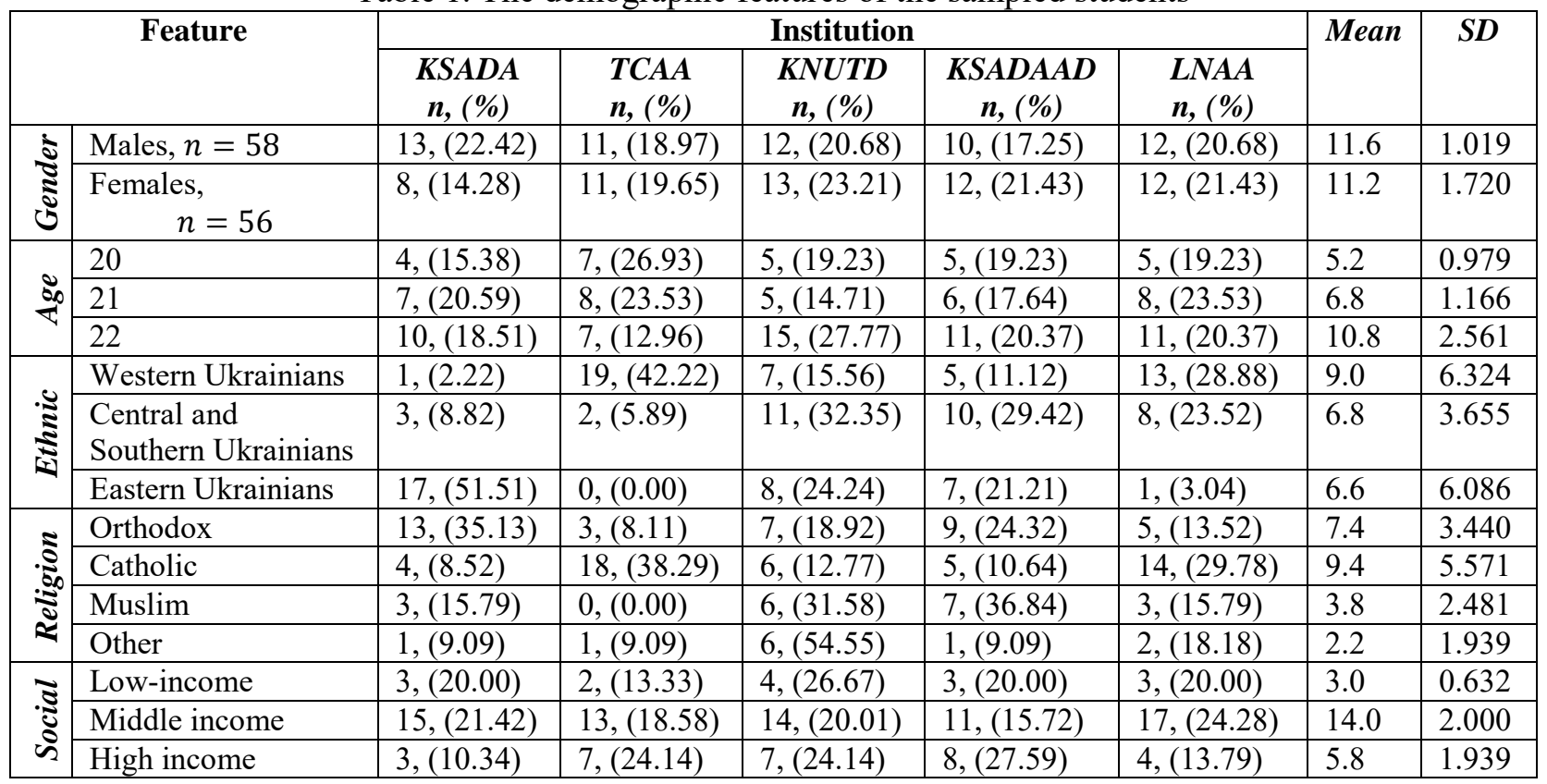

\subsection{Ethical Considerations}

The ethical considerations were addressed via the voluntary participation of the testees in the pilot study, anonymous testing without collecting the respondents' names and surnames (the participants were given a testee code). The participants were not forced to provide information about their social status or ethnic origin, however, the tests that did not provide these data were eliminated from the study. The questions for the test were formulated in a way so that offensive, bullying, or discriminatory language was avoided. The confidentiality of the research data related to the ethnic and social status of the respondents was ensured.

\subsection{Instruments}

The test on arts (can be accessed via the link https://forms.gle/KGTV8wrYcnPDRQ3T7) was specifically designed for student designers to cover the principles of design, principles of art, and
Ukrainian art (miscellaneous). The test was intended to test students' knowledge in Chromatics, Fundamentals of shaping, Layout and Composition in graphic design, principles of Art and Ukrainian folk arts and crafts, and famous artists. The test consisted of 34 dichotomously scored and multiplechoice questions that were divided into three sections (sub-domains). The first section entitled The Principles of Design included 12 questions. The second section entitled the Principles of Art comprised 12 questions. The third section entitled Ukrainian art consisted of 10 questions.

\section{Results}

The results of a one-factor CFA model in which each section is considered to be a factor such as the entire test, ethnic groups, and social groups are presented in Table 2. As can be seen in Table 2, the CFA results show the unidimensionality of the test.

Table 2. The results of a one-factor CFA model applied to the test distributed by ethnic groups and social

\begin{tabular}{|l|c|c|c|c|l|l|l|}
\hline \multicolumn{1}{|c|}{ Group } & $\chi^{\mathbf{2}}$ & CFI & TLI & RMSEA & 90\% for RMEA & df \\
\cline { 5 - 7 } & & & & & LL & UL & \\
\hline Ethnic groups & 126.124 & 0.974 & 0.972 & 0.027 & 0.026 & 0.028 & 112 \\
\hline Social groups & 127.201 & 0.953 & 0.959 & 0.027 & 0.026 & 0.028 & 112 \\
\hline
\end{tabular}




\begin{tabular}{|l|l|l|l|l|l|l|l|}
\hline All & 134.119 & 0.965 & 0.967 & 0.031 & 0.029 & 0.032 & 112 \\
\hline
\end{tabular}

The values for CFI and TLI that are $>0.95$ show a good fit between the model and data for every separate factor [21]. The RMSEA values for the groups and the entire test are lower than 0.06 (reference value) with a 95\% confidence interval which also proves a good fit for group factors.

The Chi-square values $\left(\chi^{2}\right)$ are lower than the critical value of 137.701 [22] which are expected to be lower and this indicated that there is sufficient evidence to state that there is a relationship between the test data and ethnic and social groups. Overall, the results presented in Table 2 show that the onefactor CFA model illustrates a good fit to the data and the test can be regarded as unidimensional.

The descriptive statistics and coefficients of reliability that were drawn from the whole test and each section are presented in Table 3. The Cronbach's reliability coefficient and composite reliability coefficients with factor loadings based on CFA were computed to ensure more reliable results.

Table 3. The descriptive statistics and coefficients of reliability based on the Cronbach's reliability coefficient and composite reliability coefficients and drawn from ethnic groups and social groups

\begin{tabular}{|l|l|l|l|l|}
\hline Test section & \multicolumn{1}{|c|}{ Mean } & \multicolumn{1}{c|}{$\boldsymbol{S D}$} & Cronbach $\boldsymbol{\alpha}$ & $\boldsymbol{r}$ \\
\hline PD & 8.83 & 2.11 & 0.87 & 0.951 \\
\hline PA & 8.55 & 2.06 & 0.91 & 0.821 \\
\hline UA & 8.11 & 2.25 & 0.83 & 0.781 \\
\hline ALL & 25.22 & 4.56 & 0.94 & 0.913 \\
\hline
\end{tabular}

Note: PD - Principles of Design, PA - Principles of Art, UA - Ukrainian art (miscellaneous).

As can be seen in Table 3, the values for Cronbach $\alpha$ and composite reliability coefficients with factor loadings are sufficiently high for the whole test with $\alpha=0.94$ for the whole test and $r=0.913$ for the composite reliability coefficients, respectively. The statistics show that the difference between the coefficients is negligibly small which proves the unidimensionality of the test.
The DIF results drawn from the whole test are presented in Table 4 and these are unrelated to the test sections. The items are abbreviated with 'PD' standing for Principles of Design, 'PA' standing for Principles of Art, and UA standing Ukrainian art (miscellaneous). The DIF values in the second column are obtained from Assess.ai software.

Table 4. Results of DIF computation drawn from the whole test using Assess.ai software

\begin{tabular}{|l|l|l|l|l|l|}
\hline \multicolumn{1}{|c|}{ Item } & \multicolumn{1}{c|}{ DIF } & \multicolumn{1}{c|}{ p-value } & \multicolumn{1}{c|}{ Item } & \multicolumn{1}{c|}{ DIF } & p-value \\
\hline PD1 & 0.033 & 0.979 & PA6 & 0.322 & 0.685 \\
\hline PD2 & 0.873 & 0.656 & PA7 & 4.471 & 0.159 \\
\hline PD3 & 3.217 & 0.109 & PA8 & 5.921 & 0.091 \\
\hline PD4 & 1.276 & 0.481 & PA9 & 6.578 & 0.032 \\
\hline PD5 & 4.617 & 0.117 & PA10 & 7.943 & 0.271 \\
\hline PD6 & 1.497 & 0.439 & PA11 & 8.832 & 0.128 \\
\hline PD7 & 2.298 & 0.391 & PA12 & 5.891 & 1.291 \\
\hline PD8 & 0.791 & 0.872 & UA1 & 12.795 & 0.145 \\
\hline PD9 & 0.041 & 0.873 & UA2 & 13.021 & 0.018 \\
\hline PD10 & 1.881 & 0.492 & UA3 & 12.747 & 0.023 \\
\hline PD11 & 2.561 & 0.288 & UA4 & 27.242 & 0.000 \\
\hline PD12 & 1.379 & 0.543 & UA5 & 21.438 & 0.000 \\
\hline PA1 & 4.432 & 0.192 & UA6 & 11.043 & 0.012 \\
\hline PA2 & 2.947 & 0.747 & UA7 & 8.654 & 0.048 \\
\hline PA3 & 3.937 & 0.419 & UA8 & 8.885 & 0.034 \\
\hline PA4 & 2.442 & 0.387 & UA9 & 7.579 & 0.019 \\
\hline PA5 & 2.464 & 0.358 & UA10 & 17.229 & 0.003 \\
\hline
\end{tabular}

As can be noticed in Table 4, the values seven items (UA1, UA2, UA3, UA4, UA5, UA6, UA10) in the test section entitled Ukrainian art (miscellaneous) these are highlighted bold -substantially exceeded the reference DIF detection threshold of 9.20, particularly UA4, UA5, and UA10. The Mean value 
for the other four items was 12.489 and this tended to be close to the DIF reference value. It is noteworthy that all seven items belong to the Ukrainian culture block.

Each detected DIF item is illustrated in Figure 1 providing item characteristic curves (ICCs) for the focal (ethnic groups) and reference (social status) groups. The straight line is used to illustrate the focal groups, and the dotted line stands for the reference groups. Both lines are used to clarify the probability of giving the correct answer by the testees from different ethnic and social groups. The coloured space between the lines shows the extend of the DIF effect.

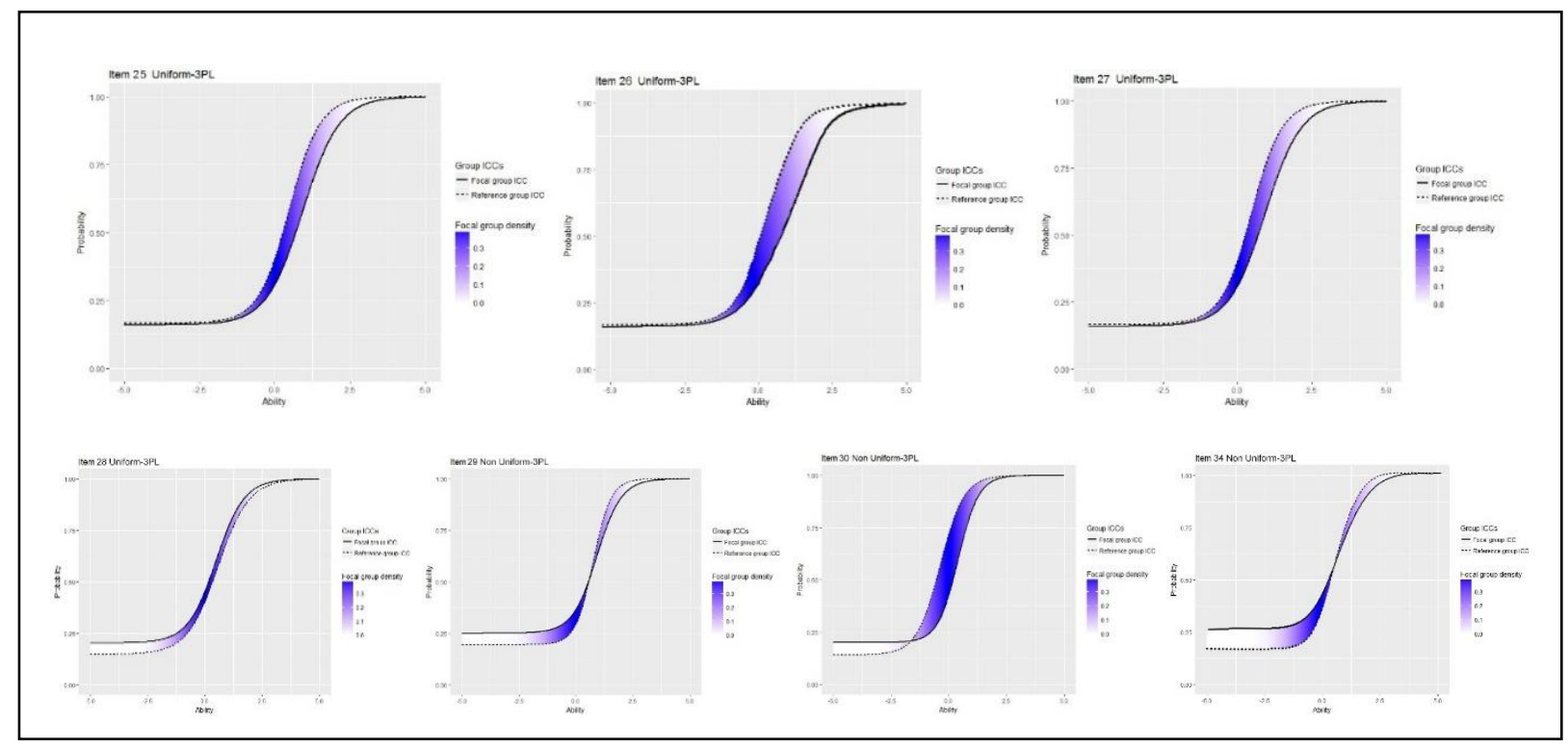

Fig. 1: ICCs for DIF items of the test in focal and reference groups

The DIF results drawn from the analysis of the test sections (principles of design, principles of art, and Ukrainian art (miscellaneous)) separately are provided in Table 5. The DIF results were obtained using the Assess.ai software.

Table 5. DIF results obtained using the Assess.ai software to analyse test sections separately

\begin{tabular}{|l|c|l|l|l|l|l|l|l|}
\hline \multicolumn{3}{|c|}{ Principles of design } & \multicolumn{3}{c|}{ Principles of art } & \multicolumn{3}{|c|}{ Ukrainian art (miscellaneous) } \\
\hline Item & DIF & $\boldsymbol{p}-$ value & Item & DIF & $\boldsymbol{p}-$ value & Item & DIF & $\boldsymbol{p}-$ value \\
\hline PD1 & 0.471 & 0.432 & PA1 & 5.222 & 0.128 & UA1 & 14.522 & 0.485 \\
\hline PD2 & 0.791 & 0.519 & PA2 & 3.741 & 0.438 & UA2 & 15.887 & 0.049 \\
\hline PD3 & 4.172 & 0.086 & PA3 & 4.351 & 0.697 & UA3 & 13.229 & 0.019 \\
\hline PD4 & 1.224 & 0.467 & PA4 & 3.239 & 0.575 & UA4 & 26.873 & 0.003 \\
\hline PD5 & 4.455 & 0.104 & PA5 & 1.659 & 0.499 & UA5 & 23.963 & 0.001 \\
\hline PD6 & 1.976 & 0.275 & PA6 & 0.489 & 0.734 & UA6 & 12.227 & 0.048 \\
\hline PD7 & 2.854 & 0.515 & PA7 & 7.781 & 0.589 & UA7 & 8.669 & 0.092 \\
\hline PD8 & 1.072 & 0.526 & PA8 & 7.557 & 0.081 & UA8 & 8.982 & 0.219 \\
\hline PD9 & 0.067 & 0.638 & PA9 & 5.678 & 0.079 & UA9 & 6.922 & 0.177 \\
\hline PD10 & 1.717 & 0.529 & PA10 & 8.112 & 0.794 & UA10 & 16.775 & 0.011 \\
\hline PD11 & 2.916 & 0.445 & PA11 & 6.275 & 0.997 & & & \\
\hline PD12 & 1.769 & 0.437 & PA12 & 6.188 & 1.679 & & & \\
\hline
\end{tabular}

The DIF values provided in Table 5 shows that the items from the Ukrainian art (miscellaneous) section such as 'UA1', 'UA2', 'UA3', 'UA4', 'UA5', 'UA6', and 'UA10' were detected as DIF. It was noteworthy that no items from two other sections were detected as DIF. There were three more items in the test (PA10 $=8.112, \mathrm{UA} 7=8.669$, UA8 $=8.982$ ) whose values were close to the DIF detection reference value of 9.20 .

The above was followed by the application of the Mantel-Haensze1/Liu-Agresti DTF method to examine DIF at the test level which includes the variance estimates $\left(t^{\wedge} 2\right)$, weighted variance estimates (Weighted $\mathrm{t}^{\wedge} 2$ ), standard errors (SE), and 
Table 6) [18].

Table 6. Results of the use of a DTF method to examine DIF at test level

\begin{tabular}{|l|l|l|l|l|}
\hline \multicolumn{1}{|c|}{ Test section } & \multicolumn{2}{c|}{ Variance estimates } & \multicolumn{1}{c|}{$\boldsymbol{S E}$} & $\boldsymbol{Z}$ \\
\hline \multirow{2}{*}{ Principles of design } & \multicolumn{1}{|c|}{$t^{2}$} & 0.047 & 0.016 & 2.751 \\
\cline { 2 - 5 } & Weighted $t^{2}$ & 0.04 & 0.013 & 2.558 \\
\hline \multirow{2}{*}{ Principles of art } & $t^{2}$ & 0.063 & 0.018 & 4.266 \\
\cline { 2 - 5 } & Weighted $t^{2}$ & 0.06 & 0.013 & 4.011 \\
\hline \multirow{2}{*}{$\begin{array}{c}\text { Ukrainian art } \\
\text { (miscellaneous) }\end{array}$} & $t^{2}$ & 0.098 & 0.041 & 3.015 \\
\hline \multirow{2}{*}{ Test (integrated) } & Weighted $\mathrm{t}^{2}$ & 0.078 & 0.032 & 3.002 \\
\cline { 2 - 5 } & $t^{2}$ & 0.063 & 0.017 & 5.375 \\
\cline { 2 - 5 } & Weighted $t^{2}$ & 0.06 & 0.01 & 6.000 \\
\hline
\end{tabular}

The values provided in Table 6 imply that the DTF variance $\left(\mathrm{t}^{\wedge} 2\right)$ for the whole test is $<0.07$ $\left(t^{\wedge} 2=0.063\right)$ which means a small DTF effect. The above results suggested that the scores for the test did not function differently at the test level in both ethnic and social status groups. It meant that the result of the test could be considered fair.

Though there were detected seven items with the DIF for the whole test, the DTF measurements showed that the DIF effect eliminated each out at test level which is related to the fact the certain ethnic groups performed better in answering some specific test items, while certain status groups performed better in answering some other test items. It is noteworthy that DTF for the Ukrainian art (miscellaneous) section was between 0.07 and 0.14 meaning a moderate DTF effect. However, the DTF variance values for the sections of principles of design and principles of art were lower than 0.07 meaning a small effect. Therefore, it could be concluded that small DTF effects found in both the whole test and each test section separately indicated that DIF effects eliminate each other at the test level.

\section{Discussion}

The attempted to address the research questions such as whether the factorial structure of the test on arts consisting of cultural questions meets the assumption of unidimensionality before the DIF method is used; whether items of the test on arts consisting of cultural questions function differently across ethnic and social groups; whether the distribution of DIF items across the cultural subdomain is different; whether the entire test scores of the test show differential test functioning (DTF) across ethnic and social groups when each domain is treated as a separate test. The strength of the study is in an attempt to address the issue of the test bias for student graphic designers in Ukraine as well as in the international institutions in other countries.
It was found that the values for CFI and TLI that are $>0.95$ showed a good fit between the model and data for every separate factor [21]. The RMSEA values for the groups and the entire test were lower than 0.06 (reference value) with a $95 \%$ confidence interval which also proved a good fit for group factors.

The Chi-square values $\left(\chi^{2}\right)$ were lower than the critical value of 137.701 which were expected to be lower and this indicated that there was sufficient evidence to state that there is a relationship between the test data and ethnic and social groups. Overall, the one-factor CFA model illustrated a good fit to the data and the test could be regarded as unidimensional.

The computation of the Cronbach's reliability coefficient and composite reliability coefficients with factor loadings based on CFA showed that the values for Cronbach $\alpha$ and composite reliability coefficients with factor loadings were sufficiently high for the whole test with $\alpha=0.94$ for the whole test and $\mathrm{r}=0.913$ for the composite reliability coefficients, respectively. The statistics showed that the difference between the coefficients was negligibly small which proved that the test was unidimensional. The use of the Assess.ai software identified the values for seven DIF items (UA1, UA2, UA3, UA4, UA5, UA6, UA10) in the test section entitled Ukrainian art (miscellaneous) these are highlighted bold - substantially exceeded the reference DIF detection threshold of 9.20, particularly UA4, UA5, and UA10. The mean value for the other four items was 12.489 and this tended to be close to the DIF reference value. It was noteworthy that all seven items belong to the Ukrainian culture block. The DIF items were from the Ukrainian art (miscellaneous) section and it was noteworthy that no items from two other sections were detected as DIF. There were three more items in the test $(\mathrm{PA} 10=8.112, \mathrm{UA} 7=8.669, \mathrm{UA} 8=8.982)$ whose values were close to the DIF detection reference value of 9.20. 
The application of the Mantel-Haenszel/LiuAgresti DTF method to examine DIF at test level which includes the variance estimates (t2), weighted variance estimates (Weighted $\mathrm{t} 2$ ), standard errors (SE), and Z-scores for the whole test and each test section implied that the DTF variance ( $\mathrm{t} 2)$ for the whole test is $<0.07-(\mathrm{t} 2=0.063)$ which meant a small DTF effect. The above results suggested that the scores for the test did not function differently at the test level in both ethnic and social status groups.

The study is consistent with the previous research emphasing the importance of DIF analysis in test validation [23]. It goes with [24-25] who claimed that DIF effects could be caused by the content of the test and might take place when latent traits were manifested unintentionally. According to [26], the item bias can take place when the sample is large and the majority of the sample is favoured with certain content which leads to the unfairness of the test. The study aligns with [27] who concluded that the latent factors compensate each other when two different groups are involved.

\section{Conclusions}

The strength of the study is in an attempt to address the issue of the test bias for student graphic designers. The inclusion of local culture-related questions in the tests on arts to assess the skills of student graphic designers influences the individuals' latent traits which leads to an increase in DIF values. Though there were detected seven items with the DIF for the whole test, the DTF measurements showed that the DIF effect eliminated each out at test level which is related to the fact the certain ethnic groups performed better in answering some specific test items, while certain status groups performed better in answering some other test items. It is noteworthy that DTF for the Ukrainian art (miscellaneous) section was between 0.07 and 0.14 meaning a moderate DTF effect. However, the DTF variance values for the sections of principles of design and principles of art were lower than 0.07 meaning a small effect. Therefore, it could be concluded that small DTF effects found in both the whole test and each test section separately indicated that DIF effects eliminate each other at the test level.

\subsection{Recommendations}

The practitioners should formulate the questions in a way so that offensive, bullying, or discriminatory language was avoided. The content should be carefully selected or it should be localised taking into account the ethnic and social features of the student population. The researchers should address the issues of tolerating the influences of ethnic and social status latent factors (cultural bias) on test fairness.

\subsection{Limitations}

Sample size, sampling techniques, and involvement of one major only in the intervention can be considered the limitations of the study.

\section{Acknowledgement:}

This study would not have been possible without experts, participants, and all other people who were involved and assisted in the data collection and processing.

\section{Conflicts of Interest:}

The authors report the existing no conflict of interest related to financial gains or personal or professional considerations.

\section{References:}

[1] Kunnan, A. J., Test fairness, test bias, and DIF, Language Assessment Quarterly, Vol. 4, No. 2, 2007, pp. 109-112. https://doi.org/10.1080/15434300701375865.

[2] Kruse, A. J., Cultural bias in testing: A review of literature and implications for music education, Update: Applications of Research in Music Education, Vol. 35, No. 1, 2015, pp. 2331. https://doi.org/10.1177/8755123315576212

[3] Drasgow, F., Nye, D. C., Stark, S. and Chernyshenko, O. S., Chapter 27: Differential Item and Test Functioning, In P. Irwing, T. Booth, and D. J. Hughes (Eds.) The Wiley Handbook of Psychometric Testing: A Multidisciplinary Reference on Survey, Scale, and Test Development, Hoboken: John Wiley \& Sons Ltd., 2018, pp. 885-889. https://doi.org/10.1002/9781118489772.ch27

[4] Nugent, W. R., Understanding DIF, and DTF: Description, methods, and implications for social work research, Journal of the Society for Social Work and Research, Vol. 8, No. 2, 2017, pp. 305-334. https://doi.org/10.1086/691525.

[5] Ballangrud, R., Husebø, S. E. and Hall-Lord, M. L., Cross-cultural validation and psychometric testing of the Norwegian version of the TeamSTEPPS ${ }^{\circledR}$ teamwork perceptions questionnaire, BMC Health Services Research, Vol. 17, Art. No. 799, 2017. https://doi.org/10.1186/s12913-017-2733-y. 
[6] Stark, S., Chernyshenko, O. S. and Drasgow, F., Examining the effects of Differential Item (Functioning and Differential) Test Functioning on selection decisions: When are statistically significant effects practically important?, Journal of Applied Psychology, Vol. 89, No. 3, 2004, pp. 497-508. https://doi.org/10.1037/0021-9010.89.3.497.

[7] Tuncay, O., DIF analysis across genders for reading comprehension parts of English language achievement exam as a foreign language, Educational Research, and Reviews, Vol. 10, No.11, 2015, p. 1505-1513. https://doi.org/10.5897/err2015.2284.

[8] Yanlou, L., Hao, Y., Tao, X., Laicheng, S. and Lu, Y., A Comparison of differential item functioning detection methods in cognitive diagnostic models, Frontiers in Psychology, Vol. 10, Art. No. 1137, 2019. https://doi.org/10.3389/fpsyg.2019.01137.

[9] Meade, A., A taxonomy of effect size measures for the differential functioning of items and scales, Journal of Applied Psychology, Vol. 95, 2010, p. 728-743. http://dx.doi.org/10.1037/a0018966.

[10] Meade, A. and Wright, N., Solving the measurement invariance anchor item problem initem response theory, Journal of Applied Psychology, Vol. 97, No. 5, 2012, p. 1016-1031. https://doi.org/10.1037/a0027934.

[11] Finch, W. H., and French, B. F., Item Response Theory (IRT), In Educational and Psychological Measurement, New York: Routledge, 2018, pp. 235-276.

[12] Hambleton, R. K., and Zhao, Y., Item Response Theory (IRT) Models for Dichotomous Data, in Wiley StatsRef: Statistics Reference Online, Hoboken, NJ: John Wiley \& Sons, Ltd., 2014.

[13] Embretsen, S. and Reise, S., Item response theory for psychologists. New York, NY: Psychology Press, 2000.

[14] Bonifay, W., Unidimensional item response theory," In Multidimensional item response theory, Newbury Park, CA: SAGE Publications, Inc, 2020, pp. 5-26.

[15] Carlson, J. E., Multidimensional Item Response Theory Models, In Introduction to Item Response Theory Models and Applications, London: Routledge, 2020, pp. 101-119.

[16] Green, B. F., Book review of Educational measurement by Brennan (Ed.), Journal of Educational Measurement, Vol. 45, No. 2, 2008, pp. 195-200. https://doi.org/10.1111/j.17453984.2008.00060.x
[17] Fidalgo, A. M., and Madeira, J. M., Generalized Mantel-Haenszel Methods for Differential Item Functioning Detection, Educational and Psychological Measurement, Vol. 68, No. 6, 2008, pp. 940-958. https://doi.org/10.1177/0013164408315265

[18] Penfield, R., DIFAS 5.0: Differential item functions analysis system. User's manual, 2013. Available online: https://soe.uncg.edu/wpcontent/uploads/2015/12/DIFASManual_V5.pdf (accessed 28.06.2021).

[19] French, B. F., and Finch, W. H., Extensions of the Mantel-Haenszel for multilevel DIF detection, Educational and Psychological Measurement, Vol. 73, 2013, pp. 648-671. https://doi.org/10.1177\%2F0013164412472341

[20] Hox, J. J. and Maas, C. J. M., The accuracy of multilevel structural equation modelling with pseudobalanced groups and small samples, Structured Equation Modelling: A Multidisciplinary Journal, Vol. 8, 2009, pp. 157-174. https://doi.org/10.1207/S15328007SEM0802_1.

[21] Cho, G., Hwang, H., Sarstedt, M. and Ringle, C. M., Cutoff criteria for overall model fit indexes in the generalized structured component analysis, Journal of Marketing Analytics, Vol. 8, 2020, pp. 189-202. https://doi.org/10.1057/s41270-020-00089-1

[22] Zach, Chi-square Distribution Table, Statology, 2018. Available online: https://www.statology.org/chi-squaredistribution-table/ (accessed 28.06.2021).

[23] Hope, D., Adamson, K., McManus, I. C., Chris, L. and Elder, A., Using differential item functioning to evaluate potential bias in high stakes postgraduate knowledge-based assessment, BMC Medical Education, Vol. 18, 2018, p. 1-7. https://doi.org/10.1186/s12909018-1143-0

[24] Ercikan, K., Arim, R., Law, D., Domene, J., Gagnon F., and Lacroix S., Application of thinkaloud protocols for examining and confirming sources of differential item functioning identified by expert reviews, Educational Measurement: Issues and Practice, Vol. 29, 2010, p. 24-35. https://doi.org/10.1111/j.17453992.2010.00173.x

[25] Martinková, P., Drabinová, A., Liaw, Y., Sanders, E. A., McFarland, J. L. and Price, R. M., Checking Equity: Why Differential Item Functioning Analysis Should Be a Routine Part of Developing Conceptual Assessments, $C B E$ Life Science of Education, Vol. 16, No. 2, 2017, 
pp. 1-13. https://doi.org/10.1187/cbe.16-100307

[26] Zhu, X. and Aryadoust, V., An investigation of mother tongue differential item functioning in a high-stakes computerized academic reading test, Computer Assisted Language Learning, Vol. 33, 2020 , p. $1-24$. https://doi.org/10.1080/09588221.2019.1704788

[27] Ozdemir, B. and Alshamrani, A. H., Examining the Fairness of Language Test Across Gender with IRT-based Differential Item and Test Functioning Methods, International Journal of Learning, Teaching and Educational Research, Vol. 19, No. 6, 2020, p. 27-45. https://doi.org/10.26803/ijlter.19.6.2.

Contribution of Individual Authors to the Creation of a Scientific Article (Ghostwriting Policy)

Marianna Nerozna: concept and design, critical revision of manuscript.

Ievgen Gula: data acquisition, data analysis and interpretation, drafting manuscript.

Mykhailo Rudenko: data acquisition, data analysis and interpretation, drafting manuscript.

Oksana Maznichenko: drafting manuscript, critical revision of manuscript.

Oleksandra P. Basanec: data acquisition, data analysis, drafting manuscript.

Volodymyr Prusak: supervision, critical revision of manuscript, final approval.

\section{Sources of Funding for Research Presented in a Scientific Article or Scientific Article Itself}

There is no source of funding of this research

Creative Commons Attribution License 4.0

(Attribution 4.0 International, CC BY 4.0)

This article is published under the terms of the Creative Commons Attribution License 4.0

https://creativecommons.org/licenses/by/4.0/deed.en US 